\title{
Oncology Health Care Providers' Perceptions of the Psychological Impact of COVID-19 on Oncology Patients
}

Melody Nichole Chavez ( $\sim$ mnchavez@usf.edu )

University of South Florida College of Public Health https://orcid.org/0000-0002-8707-7072

Victoria K. Marshall

University of South Florida College of Nursing

Dinorah Martinez Tyson

University of South Florida College of Public Health

Tina M. Mason

University of South Florida College of Nursing

Kaitlyn Rechenberg

University of South Florida College of Nursing

Research Article

Keywords: Cancer, COVID-19, coronavirus, pandemic, depression, anxiety

Posted Date: April 5th, 2021

DOI: https://doi.org/10.21203/rs.3.rs-331588/v1

License: (9) This work is licensed under a Creative Commons Attribution 4.0 International License.

Read Full License 


\section{Abstract}

Purpose. The COVID-19 pandemic and social isolation measures have had a profound impact on the psychological and mental well-being of people living with cancer. The aim of this study was to explore oncology health care professionals' (OHCP) perceptions of psychological effects of COVID-19 among people in active cancer treatment.

Methods. An exploratory qualitative study using semi-structured interviews with a purposive sample of $\mathrm{OHCP}$, who were actively participating and providing care to patients with cancer and undergoing treatment. Interviews were audio-recorded, transcribed, and coded using Atlas.ti v8. Inductive applied thematic analysis techniques were employed to identify emergent themes.

Results. A total of $30 \mathrm{OHCP}$ participated in the study with the majority being registered nurses $(70 \%)$, worked in the outpatient oncology clinic (56.7\%) and worked in current position 1 - 5 years $(53.3 \%)$. The overarching themes included: (1) cancer care treatment disrupted due to patients fear of exposure to COVID-19; (2) social distancing restrictions had a negative impact on social support and supportive services exacerbating psychological and physical well-being among oncology patients; (3) pandemicrelated stressors lead to overwhelmed coping skills among oncology patients; and (4) OHCP play vital roles in providing emotional support to comfort, reassure and connect oncology patients with their family/friends through technology.

Conclusion. Our findings highlight increased psychological distress during the pandemic. Behavioral health interventions for oncology patients should focus within the scope of the "new world of Covid-19" of reduced face to face support and increased online support.

\section{Introduction}

People living with cancer (PLWC) experience considerable psychological and emotional distress following a cancer diagnosis and treatment [1]. Compared to the general population, PLWC are at higher risk for developing mental health disorders [2]. It is estimated that $29-43 \%$ of the cancer population meets the diagnostic criteria for a mental health disorder such as anxiety, depression and stress-related conditions such as post-traumatic stress disorder [3]. Left untreated, such disorders can negatively affect cancer treatment compliance, symptom control, hospitalization period, recovery, and quality of life $[4,5]$.

The COVID-19 pandemic has had a profound impact on the psychological and mental well-being of individuals [1]. While cancer patients are already uncertain about their future and managing their cancer care, recent literature has showcased psychosocial problems and needs of cancer patients as increasing due to the pandemic. Lui et al. (2020) found that the prevalence of psychological problems increased from $36.95 \%$ to $43.8 \%$ and among cancer patients in China since the outbreak of COVID-19 [6]. Social isolation measures to contain the spread of the infection caused cancer treatment delays which can cause distress about potential negative outcomes [7]. Sud et al., (2020) identified modest delays of three and six months in cancer surgery will significantly impact survival [8]. In addition, changes to social 
support systems have caused unintended consequences for mental health such as increased risk of isolation and loneliness, anxiety, and depression. These factors are independently associated with misuse of substances as well as self-harm [9].

Social support is vital to PLWC and has been shown to be a protective factor against adverse psychological effects [3]. Interactions with physicians, nurses and other health care staff play a valuable role in the delivery of support, especially to those in active cancer treatment [10]. Furthermore, OHCP promote strategies to reinforce existing social support such as encouraging connections with family and friends, fostering cancer support groups, and advocating for resources to enhance coping mechanisms through behavioral health interventions[10].

Understanding the perceptions among OHCP regarding the psychological impact the virus is having on PLWC is vital as they are responsible for identifying the psychosocial health needs of this vulnerable population. Limited research has explored this phenomenon through the viewpoints of OHCP, who can provide a better understanding of the various dimensions that COVID-19 is inflecting on PLWC. Therefore, the purpose of this study was to explore (OHCP) perceptions of psychological effects of cancer and COVID-19 among PWC.

\section{Methods}

\section{Study Design}

This study used an exploratory qualitative research design, which allowed for open-ended questions to obtain an in-depth understanding of the perception of the psychological impact of the pandemic on patients in active cancer treatment from OHCP perceptions [11]. This study was approved by the Blinded for Review and the Blinded for Review.

\section{Recruitment}

Purposeful sampling techniques, which are appropriate for exploratory research, were utilized to recruit OHCP with different backgrounds and views to provide in-depth information about the psychological impact of COVID-19 experienced by their patients [11]. Eligibility included OHCP who were actively practicing and providing care to patients diagnosed with cancer and undergoing treatment. An IRBapproved flyer was distributed to recruit OHCP through established partnerships through local oncology facilities, professional organizations, community-based organizations, social media, and email Listservs. Interested OHCP were provided with the Principal Investigator (PI) email to ask questions and express willingness to participate. Telephone interviews were conducted at a time that was convenient to eligible OHCP [12]. Informed consent was obtained prior to data collection. Recruitment continued until data saturation was achieved. Data saturation was considered the point at which no new themes arose from the data [13].

\section{Data Collection Procedures}


Interviews were conducted by the PI (VM) via the telephone to abide by social distancing regulations. Demographic, professional and employment information were collected. All interviews were undertaken between May through July of 2020. Interviews were audio-recorded with informed consent, transcribed verbatim by a professional company and de-identified with participant-assigned pseudonyms. Transcripts were uploaded into Atlas.ti v.8 for data management and analysis [14]. To compensate for their time, participants received a $\$ 20$ gift card.

\section{Data Analysis}

Thematic analysis was undertaken to analyze the data using an iterative process where transcripts were independently read by study team members (VM, MC, DMT, and TM) [13]. Preliminary review of the transcripts and initial coding was completed by VM, MC, DMT, and TM to develop a codebook that included a priori and emergent codes with corresponding definitions. A subsample of transcripts $(n=3)$ were reviewed and double coded to assess intercoder reliability. Intercoder reliability was evaluated using Cohen's kappa which was assessed at .72, indicating substantial agreement between raters (VM and MC) [15]. All transcripts were then coded by one coder (MC) and PI (VM) coded random transcripts to ensure reliability of coding. In addition, the survey data was coded and uploaded into SPSS v24 [16] to provide descriptive statistics of participants and summarize professional and employment measures.

\section{Results}

Overall, the interview sample was comprised of a total of 30 participants (Table 1). The greatest proportion of participants represented registered nurses (70\%), followed by advanced practice registered nurses (13.3\%). The majority (56.7\%) worked in the outpatient oncology clinic and $53.3 \%$ worked in their current position 1-5 years. Four themes emerged from the qualitative interviews.

\section{Theme 1: Cancer care treatment disrupted due to patients fear of exposure to COVID-19}

The most voiced impact of COVID-19 reported by OHCP was the increase of fear among their cancer patients. Patients seemed well aware of their immunocompromised system, placing them at greater risk for contracting the virus and resulting in poorer outcomes such as death. This caused them to delay or cancel their treatment including chemotherapy infusion, radiation therapy, follow-up care, annual cancer screening, and cancer-related surgeries. Providers reported that patients sought out different modalities of cancer treatment such as taking oral chemotherapy to decrease their hospital visits. For example, a registered nurse (Participant \#1) noted patients are panicking asking questions such as "If I get this, am I gonna die? Should I skip my chemotherapy?" Another registered nurse (Participant \#27) reported that fear has resulted in some patients "not telling their oncologists what is actually going on" with their health to prevent having to come to the hospital. OHCP reported cancer patients were also caregivers to family members with compromised systems leading them to delay their treatment. This was illustrated by a genetic counselor (Participant \#5) who stated, "They were fearful of not only getting the virus themselves, but also taking it home and giving it to their elderly parents." Finally, the fear surrounding the 
virus and delay in treatments resulted in several OHCP feeling unsure of how much PLWC will be affected long-term in regard to cancer progression.

\section{Theme 2: Social distancing restrictions had a negative impact on social support and supportive services exacerbating psychological and physical well-being among patients}

\section{Subtheme: No visitor policy impacting the psychological well-being of patients}

As a result of social distancing measures, the implementation of limiting or no visitors' policies meant that cancer patients had to receive a cancer diagnosis, undergo treatment, and surgical procedures without a family member or caregiver present. OHCP reported substantial mental distress among cancer patients not being able to have loved ones present with them at the hospital or oncology clinic. This was especially challenging among patients who required lengthy stays such as those receiving a blood and marrow transplant. A registered nurse (Participant \#15) discussed many of her patients expressed, "adding the no visitors [policy] will just put me over the edge. It's essentially life or death." Many OHCP reported the lack of visitors and inability to leave the hospital unit caused them to feel cooped up, anxious, and frustrated. An inpatient registered nurse working on the blood and marrow transplant unit (Participant \#25) stated, "I actually had a patient sign out AMA yesterday, for the first time in four and a half years." Some facilities allowed one caregiver to stay with the patient; however, they were not allowed to leave the unit or hospital. For patients who often had family members present during chemotherapy or providers' appointments, they were now required to wait in their cars.

Some OHCP discussed challenges they faced in providing patient education as hospital and clinic policies limited providers' contact and discussion with their patients. This was difficult for newly diagnosed patients as an advanced practice registered nurse (Participant \#5) explained, "If you have patients that need a lot of discussion, we're told to limit contact. They don't have a second set of ears. They might need you to repeat things....Everything looks and feels different." Lastly, end-of-life care was especially challenging for both OHCP and their patients. Many patients were taken off life support without family present. A registered nurse (Participant \#3) spoke about a leukemia patient who contracted COVID-19, describing his final moments, "We had his wife talk to him on the phone before he was intubated, he declined pretty rapidly, and he died a couple of days later. We had to Zoom his family in for the withdrawal of care.... it was pretty emotional."

\section{Subtheme: Suspension of cancer support services impacted patients striving to continue cancer treatment}

Cancer support services and resources, such as support groups and housing/lodging services to help and assist PLWC were discontinued, further exacerbating their emotional well-being. For example, cancer patients who did not own a car or were unable to drive, found that transportation services, such as the American Cancer Society Road to Recovery, were discontinued to protect their volunteers. Alternative transportation options were also discussed, however there was a concern of increased exposure to the virus for the patient by using public transportation. This was illustrated by a licensed mental health 
counselor (Participant \# 4) who stated, "Transportation is a problem...It can get very expensive using Uber or a taxi... Plus, then, it also puts the cancer patient at risk because they don't know who's been in and out of the car, and who they're riding with." For patients who lived out of town/state and required lodging, they faced challenges in continuing with their treatment. This was described by a registered nurse who stated, "American Cancer Society program [Hopes Lodge], a lodging program was not in use...finding a hotel to stay overnight if they have treatment two different days... There are issues with that."

Cancer support groups, which were often recommended by OHCP who identified patients at risk for depression or loneliness, were also suspended. Activities at the local gym or pool were no longer options to encourage patients to engage in physical wellness and to keep busy. Several OHCPs discussed challenges social workers faced with providing resources for patients who required meals and medications delivered to their homes. Other services as discussed by an advanced practice registered nurse (Participant \# 5) reported "patients couldn't get wigs or prosthetics," which had the potential to affect the cancer patient's ability to retain or regain their independence or feel empowered about themselves. For cancer patients with chronic pain, an advance practice registered nurse (Participant \#9) stated, "we couldn't get physical therapy. No aqua therapy. No massage therapy. No acupuncture. These kinds of complementary integrative physical kinds of measures that people found helpful..."

\section{Theme 3: Pandemic-related stressors overwhelmed patients and affected their coping skills}

The relationship between pandemic-related stress and coping strategies, represented a critical factor in the care of PLWC. The majority of OHCP discussed a significant increase of patients with depression and anxiety exacerbated by stressors brought on by the pandemic. However, an advanced practice registered nurse (Participant \#9) working in a pain clinic discussed several unique scenarios of cancer patients misusing pain medication as a means to cope. She described, "about a third of the patients I care for have either substance use disorder, past or present, or maybe troubling misuse of the substances, or mental health challenges, and so a lot of patients are experiencing exacerbations of their mental health issues and taking too much pain med." She also discussed the transparency of some of her patients requesting early refills on medication by stating, "They'll just write in a MyChart message, 'My kids are driving me crazy. I don't think I can take this being cooped up anymore. I need an early refill of my morphine."'

In addition, several OHCPs discussed situations of cancer patients calling and expressing suicide ideations or threatening suicide. For example, a registered nurse (Participant \#12) stated, "We've actually had a few, probably say, half a dozen that I can think of, that during this whole time have called me and threatened suicide." Another registered nurse (\#23), stated "We have patients that express ideation for suicide, "I want out so that I can end it all." She went on to describe an episode of a cancer patient leaving a self-harming message on the patient portal stating, "as soon as that was seen, which is usually within an hour or two, but that's a long time if somebody is in distress."

Theme 4: During the pandemic OHCP played a vital role in providing emotional support to comfort, reassure and connect patients with their family/friends through technology 
Due to the increase of emotional distress and lack of social and supportive services, OHCP discussed a variety of strategies employed to help their patients. The majority of the nurses described how they assisted their patients in utilizing technologies such as Zoom and FaceTime, to stay connected with family and friends, especially older patients who were less familiar with such platforms. A registered nurse (Participant \#20) described how nursing staff are "just trying to make it easier for them, take their mind off things." For cancer patients receiving bad news, while it was not ideal, technology kept family connected virtually. This was described by a registered nurse (Participant \#28), "they [PLWC] have to be alone and their family member via Facetime or on a phone call can listen in."

In addition to assisting with technology, nurses also reported spending more time than usual with patients and proving emotional and physical support. This was especially challenging from a surgical standpoint as an advanced practice registered nurse (Participant \#17) discussed, "not being able to have someone with them I think is the hardest thing, not only for them, but for myself to be able to explain to them that, you know, we are here for them, as physically and as emotionally as possible." OHCP went above and beyond to help lessen their patients' mental anguish. For example, a registered nurse (Participant \#23) explained "You have to put a few more minutes in with every patient. Really talk them through things, encourage them." Other OHCP discussed making telephone appointments to connect and check in with their patients. A licensed mental health counselor (Participant \#10), discussed, "I've also encouraged them if they're having a bad day or a bad moment and they need to reach out to somebody, to also call, and I try to call them back in-betweens scheduled appointments, just to really have that connection."

\section{Discussion}

We found that oncology patients are faced with a number of challenges during the pandemic that can negatively impact their mental well-being. The American Society of Clinical Oncology (ASCO) has reported similar findings, with nearly $45 \%$ of cancer patients noting a negative impact of their mental wellbeing due to COVID-19 [17]. Moreover, similar findings have been reported among $22.8 \%$ of chronic disease patients that reported abnormal psychological impact due to COVID-19 [18]. The increased fear of exposure to COVID-19 among PLWC was a prominent finding reported among the OHCP in the study. Given high levels of distress during the pandemic observed in the general population, PLWC are more vulnerable to the virus due to immunosuppression [19]. Furthermore, their outcomes are worse with a higher mortality rate than the general population [20]. These findings align with recent literature which collected the experiences of patients with cancer and revealed that $55 \%$ of patients worried about contracting COVID-19 infection and $26 \%$ had concerns regarding the disruption of their cancer treatment due to the virus [21]. We speculate that the uncertainty of their cancer care also contributes to the psychological distress for fear of their cancer progressing requiring more complex care later. In a study among young adults with cancer, postponed and canceled treatments due to the pandemic increased anxiety symptoms due to the uncertainty of the status of their cancer [22]. 
While social distancing policies in the United States were adopted to limit the spread of the virus, such restrictions had a negative impact for PLWC psychological well-being. Social support following a cancer diagnosis has been well documented to decrease the experience of psychological distress, improve emotional well-being [23] and positively adjust to their diagnosis and treatment [24]. OHCP reported increased levels of mental distress such as anxiety and frustration among their patients, as family members or caregivers were unable to be present with them due to limitation or no visitor policies. Such protective measures exacerbated feelings of isolation and loneliness. These findings are consistent with results from a recent study by Gallagher et al (2020) that identified people living with cancer who experienced loneliness pre-COVID were 4.5 times at higher risk for developing depression [25]. Moreover, $42 \%$ wished they had more emotional support during the pandemic [26].

As a result of the increase of emotional distress, several OHCP discussed cancer patients misuse of prescription medication as a means of coping during the pandemic. Chemical coping to reduce stress has been identified among cancer patients with higher rates of emotional distress, psychological disorders and increased pain [27]. In addition, they are prescribed higher rates of opioids placing them at risk for misuse of substances [28]. To the authors' knowledge, no studies have been conducted specifically to cancer patients and misuse of substances as a result of the pandemic, however our findings are consistent with recent literature showcasing that coronavirus could make individuals with past or present substance use disorder (SUD)more vulnerable to complications of substance use [29]. It is well known that "addiction is a disease of isolation", further exacerbating efforts to maintain social connections during a time of enormous stress and dislocation [30].

In addition, OHCP also reported concern of cancer patients who may be at increased risk of self-harm and suicide. Numerous studies have shown increased suicidal behavior during the pandemic secondary to rise in stress, anxiety, fear and loneliness in the general population [31]. This a concern as Archer et. al (2020) reported a decline in the number of patients referred to psychological services for support, advice and care showcasing cancer patients needs are not being met, especially during a crisis [7]. This also showcases how vital it is for OCHP to implement appropriate safety nets and services to protect PLWC mental health.

Numerous behavioral health interventions were discussed among OHCP. The use of online platforms such as Zoom, and FaceTime allowed for cancer patients to remain in contact with family, friends, and health care providers. OHCP also connected with patients via telephone to check on their well-being. Online platforms and telehealth services have been shown to be instrumental during this unprecedented time [32,33]. Several national organizations provide guidance on assessing and addressing psychological distress among PLWC during the pandemic. These are highlighted in Table 2 which serves as a resource for OHCP.

\section{Strengths And Limitations}


The use of qualitative research methods enabled us to explore the perceptions of the psychological implications of the pandemic among PLWC from OHCP perspectives. We feel that our results contribute to the understanding of the impact COVID-19 is placing on PLWC through the viewpoint of the providers caring from them. While our study had many strengths, limitations should be considered. We recognize that our findings are limited by the under-representation from some OHCP such behavioral health providers who screen, assess, and educate patients for common mental health and SUD. In addition, it is unknown the enrollment of racial minority patients with cancer within each of the employment settings of the OHCP. COVID-19 has revealed deep-seated inequalities in health care for communities of color [34], therefore it is important to understand how race can affect the care they receive. Finally, our study only focused on the OHCP perspective and not the oncology patient. It is unknown what other psychological challenges they may be experiencing through their life experiences during this pandemic. However, the data clearly highlights key issues that have been presented among PLWC as well as concerns and opportunities for future research and intervention.

\section{Conclusion}

Our study provides an enhanced perspective on OHCP' perceptions of psychological effects of the COVID19 pandemic among PLWC. Fear and anxiety are common among PLWC highlighting the critical need to screen more frequently for psychological distress and monitor the mental well-being as the pandemic continues. OHCP should regularly engage with patients on reliable information about mental health resources as well as tips to stay safely connected to family and friends. Interventions should focus on increasing social interaction within the scope of the "new world of COVID-19" of reduced face-to-face support and increased online support. Future research will need to be conducted to understand how PLWC have adapted to safety measures and long-term implications of their cancer in regard to their mental health status.

\section{Declarations}

Acknowledgements: This study was funded by the University of South Florida, College of Nursing Funding: This research was funded by the University of South Florida, College of Nursing (PI: Marshall)

Conflicts of interest/Competing interests: The authors have no conflict of interest or competing interest to report.

Ethics approval: This exploratory qualitative study was in accordance with the ethical standards. The Institutional Review Board (IRB) of University of South Florida and the Scientific Research Committee at the Moffitt Cancer Center approved this study.

Consent to participate: Informed consent was obtained from all individual participants included in this study. 
Consent for publication: The authors affirm that human research participants provided informed consent for publications of the data.

Availability of data and material: Data would be available upon request.

Authors' contributions: All authors contributed to the study conception and design. Victoria Marshall prepared the material collected the data and analysis. Melody Chavez assisted in analysis of data. Martinez Tyson provided feedback on the qualitative data collection and analysis as well as participated in the team debriefing meetings. Tina Mason provided material preparation, conceptualization, data analysis and review and editing of the manuscript. Kaitlyn Rechenberg assisted with methodology, table development, and the review and editing of the manuscript. The first draft of the manuscript was written by Melody Chavez. All authors commented on previous versions of the manuscript. All authors read and approved the final manuscript.

\section{References}

1. O'Hea E, Kroll-Desrosiers A, Cutillo AS, Michalak HR, Barton BA, Harralson T, et al. Impact of the mental health and dynamic referral for oncology (MHADRO) program on oncology patient outcomes, health care utilization, and health provider behaviors: a multi-site randomized control trial. Patient education and counseling. 2020;103(3):607-16.

2. Kawashima Y, Yonemoto N, Inagaki M, Inoue K, Kawanishi C, Yamada M. Interventions to prevent suicidal behavior and ideation for patients with cancer: a systematic review. General hospital psychiatry. 2019;60:98-110.

3. Niedzwiedz CL, Knifton L, Robb KA, Katikireddi SV, Smith DJ. Depression and anxiety among people living with and beyond cancer: a growing clinical and research priority. BMC cancer. 2019;19(1):1-8.

4. Pitman A, Suleman S, Hyde N, Hodgkiss A. Depression and anxiety in patients with cancer. Bmj. 2018;361.

5. Jacobsen PB. Screening for psychological distress in cancer patients: challenges and opportunities. American Society of Clinical Oncology; 2007.

6. Liu X, Liu F, Tong F, Peng W, Wen M, Zou R, et al. Psychological reactions and interventions to help Cancer patients cope during the COVID-19 pandemic in China. Journal of Psychosocial Oncology Research and Practice. 2020;2(3).

7. Archer S, Holch P, Armes J, Calman L, Foster C, Gelcich S, et al. No turning back'Psycho-oncology in the time of COVID-19-insights from a survey of UK professionals. Psycho-oncology. 2020.

8. Sud A, Jones ME, Broggio J, Loveday C, Torr B, Garrett A, et al. Collateral damage: the impact on outcomes from cancer surgery of the COVID-19 pandemic. Annals of Oncology. 2020;31(8):1065-74. 
9. Elovainio M, Hakulinen C, Pulkki-Råback L, Virtanen M, Josefsson K, Jokela M, et al. Contribution of risk factors to excess mortality in isolated and lonely individuals: an analysis of data from the UK Biobank cohort study. The Lancet Public Health. 2017;2(6):e260-e6.

10. Merluzzi TV, Philip EJ, Yang M, Heitzmann CA. Matching of received social support with need for support in adjusting to cancer and cancer survivorship. Psycho-oncology. 2016;25(6):684-90.

11. Bernard HR. Research methods in anthropology: Qualitative and quantitative approaches: Rowman \& Littlefield; 2017.

12. Novick G. Is there a bias against telephone interviews in qualitative research? Research in nursing \& health. 2008;31(4):391-8.

13. Guest G, Bunce A, Johnson L. How many interviews are enough? An experiment with data saturation and variability. Field methods. 2006;18(1):59-82.

14. Friese S. ATLAS. ti 8 Windows-Inter-Coder Agreement Analysis Copyright $\odot 2020$ by Berlin: ATLAS. ti Scientific Software Development GmbH; 2020.

15. McHugh ML. Interrater reliability: the kappa statistic. Biochemia medica. 2012;22(3):276-82.

16. Corp. I. IBM Spss Statistics for Windows, Version 24.0. In: Corp. I, editor. Armonk, NY2016.

17. Gregory B. National Cancer Opinion Survey: Cancer Screening and Mental Health During COVID-19, Racism and Cancer Care, and Misperceptions Around Cancer Clinical Trials. 2020 [Available from: https://www.cancer.net/blog/2020-10/2020-national-cancer-opinion-survey-cancer-screening-andmental-health-during-covid-19-racism-and.

18. Addis SG, Nega AD, Miretu DG. Psychological impact of COVID-19 pandemic on chronic disease patients in Dessie town government and private hospitals, Northeast Ethiopia. Diabetes \& Metabolic Syndrome: Clinical Research \& Reviews. 2021;15(1):129-35.

19. Chen DS, Mellman I. Oncology meets immunology: the cancer-immunity cycle. immunity. 2013;39(1):1-10.

20. Al-Shamsi HO, Alhazzani W, Alhuraiji A, Coomes EA, Chemaly RF, Almuhanna M, et al. A practical approach to the management of cancer patients during the novel coronavirus disease 2019 (COVID19) pandemic: an international collaborative group. The oncologist. 2020;25(6):e936.

21. Schoenmaekers JJ, Hendriks LE, van den Beuken-van MH. Palliative Care for Cancer Patients During the COVID-19 Pandemic, With Special Focus on Lung Cancer. Frontiers in Oncology. 2020;10.

22. Košir U, Loades M, Wild J, Wiedemann M, Krajnc A, Roškar S, et al. The impact of COVID-19 on the cancer care of adolescents and young adults and their well-being: Results from an online survey conducted in the early stages of the pandemic. Cancer. 2020;126(19):4414-22.

23. Applebaum AJ, Stein EM, Lord-Bessen J, Pessin H, Rosenfeld B, Breitbart W. Optimism, social support, and mental health outcomes in patients with advanced cancer. Psycho-oncology. 2014;23(3):299-306.

24. Snyder KA, Pearse W. Crisis, social support, and the family response: exploring the narratives of young breast cancer survivors. Journal of psychosocial oncology. 2010;28(4):413-31. 
25. Gallagher S, Bennett KM, Roper L. Loneliness and depression in patients with cancer during COVID19. Journal of psychosocial oncology. 2020:1-7.

26. Gregory B. Cancer Screening and Mental Health During COVID-19, Racism and Cancer Care, and Misperceptions Around Cancer Clinical Trials. 2020 [Available from: https://www.cancer.net/blog/2020-10/2020-national-cancer-opinion-survey-cancer-screening-andmental-health-during-covid-19-racism-and.

27. Jairam V, Yang DX, Verma V, James BY, Park HS. National Patterns in Prescription Opioid Use and Misuse Among Cancer Survivors in the United States. JAMA network open. 2020;3(8):e2013605-e.

28. Paice JA. Navigating Cancer Pain Management in the Midst of the Opioid Epidemic. Oncology (08909091). 2018;32(8).

29. Ornell F, Moura HF, Scherer JN, Pechansky F, Kessler FHP, von Diemen L. The COVID-19 pandemic and its impact on substance use: Implications for prevention and treatment. Psychiatry research. 2020;289:113096.

30. Roe L, Proudfoot J, Tay Wee Teck JB, Irvine RDG, Frankland S, Baldacchino AM. Isolation, solitude and social distancing for people who use drugs: an ethnographic perspective. Frontiers in Psychiatry. 2020.

31. Sher L. The impact of the COVID-19 pandemic on suicide rates. QJM: An International Journal of Medicine. 2020;113(10):707-12.

32. Lin JC, Humphries MD, Shutze WP, Aalami OO, Fischer UM, Hodgson KJ. Telemedicine platforms and their use in the coronavirus disease-19 era to deliver comprehensive vascular care. Journal of vascular surgery. 2021;73(2):392-8.

33. Ratnasekera N, Perera I, Kandapolaarachchige P, Surendra G, Dantanarayana A. Supportive care for oral cancer survivors in COVID-19 lockdown. Psycho-oncology. 2020;29(9):1409-11.

34. Abuse S, Administration MHS. Double jeopardy: COVID-19 and behavioral health disparities for Black and Latino communities in the US. 2020.

\section{Tables}

Table 1: OHCP participant characteristics 
Participant characteristic

Participant characteristic

Age (Mean, SD)

Sex

Women

Male

\section{Ethnicity}

Hispanic/Latino

Not Hispanic/Latino

\section{Race}

American Indian/Alaska Native

Asian

Native Hawaiian/Pacific Islander

Black/African American

White

Other

\section{Highest Level of Education}

Nursing Diploma

Completed Associate's Degree

Completed Bachelor's Degree

Completed Master's Degree

Completed Doctoral Degree

Completed Medical Degree

\section{Professional Title}

Registered Nurse

Advanced Practice Registered Nurse

Doctor (Oncologist)

Pharmacist

Licensed Mental Health Counselor

$3(10 \%)$
$5(16.7 \%)$

$25(83.3 \%)$

$2(6.7 \%)$

$3(10.0 \%)$

$24(80.0 \%)$

$1(3.3 \%)$

$1(3.3 \%)$

$20(66.7 \%)$

$5(16.7 \%)$

$2(6.7 \%)$

2 (3.3\%)

$21(70.0 \%)$

$4(13.3 \%)$

2 (6.7\%)

1 (3.3\%)

1 (3.3\%)
$40.80(S D=13.309)$

Oncology Health care provider $(\mathrm{N}=30)$

$$
\text { n (\%) }
$$

$27(90.0 \%)$ 


\begin{tabular}{|ll|}
\hline Participant characteristic & Oncology Health care provider $(\mathbf{N}=\mathbf{3 0})$ \\
\hline Eenetic Counselor & $1(3.3 \%)$ \\
Outpatient Oncology Clinic & $17(56.7 \%)$ \\
Inpatient Oncology Clinic & $11(36.7 \%)$ \\
Both & $2(6.7 \%)$ \\
Number of Years in Current Position & \\
Less than one year & $6(20.0 \%)$ \\
$1-5$ years & $16(53.3 \%)$ \\
$6-10$ years & $2(6.7 \%)$ \\
$11-15$ years & $2(6.7 \%)$ \\
Above 16 years & $4(13.3 \%)$ \\
Number of Hours per Week in Oncology Setting & \\
Less than 10 hours & - \\
10-20 hours & $1(3.3 \%)$ \\
$21-30$ hours & - \\
$31-40$ hours & $23(76.7 \%)$ \\
More than 40 hours & $6(20 \%)$ \\
\hline
\end{tabular}

Table 2: Resources for Oncology Patients During the COVID-19 Pandemic

Resource Description




\section{Adolescent and Young Adult Cancer Coalition}

https://www.covidayacancer.org/patient-resources
Provides patients with online classes, emotional support, financial resources, and general information about the COVID-19 pandemic and cancer.

\section{American Cancer Society}

https://www.cancer.org/about-us/what-we-do/coronaviruscovid-19-and-cancer.html
- The American Cancer Society COVID-19 and cancer guide provides the following resources for oncology patients during the pandemic: Routine medical care during the pandemic vaccines

Cancer screening and

Common questions about COVID-19 and cancer
American Society of Clinical Oncology

https://www.asco.org/asco-coronavirus-information
Provides resources for oncology patients and providers about emotional support programs, direct patient care during the pandemic, surveys, data, and publications related to COVID-19 and cancer.

\section{Association of Community Cancer Centers}

https://www.accc-cancer.org/home/news-media/coronavirusresponse
Provides webcasts, podcasts, publications, and other resource links addressing COVID-19 and cancer for patients and providers.
CancerCare

https://www.cancercare.org/coronavirus
Provides resources such as the ability to speak with a master's prepared oncology social worker, 
online support groups, online workshops, podcasts, and publications related to coronavirus.

\section{Cancer Support Community}

https://www.cancersupportcommunity.org/blog/2020/06/whatcancer-patients-survivors-and-caregivers-need
Provides access to a helpline with counselors and resource specialists, financial support information, virtual support discussion boards, radio show and other video/audio resources, and general information about COVID-19.

\section{Centers for Disease Control and Prevention}

https://www.cdc.gov/cancer/survivors/staying-well-athome.htm
Provides resources for oncology patients including how to prevent secondary illness, what to do if you start to feel symptoms of a secondary illness, and how to manage oncology care during the pandemic.

\section{European Society for Medical Oncology}

https://www.esmo.org/for-patients/patient-guides/cancer-careduring-the-covid-19-pandemic
Provides information about how COVID-19 will affect cancer care and general information about COVID-19.

\section{National Cancer Institute}

https://www.cancer.gov/about-cancer/coronavirus/coronaviruscancer-patient-information
Provides frequently asked questions and general information about COVID-19 and cancer, including information about treatment, clinical trials, coping, and general information about coronavirus.

\section{OncoLink}

https://www.oncolink.org/support/resources/coping-with-covid19
Provides information about how to manage cancer and COVID-19, how to cope with anxiety, how to stay safe, and an up-to-date list of financial resources for patients with cancer.

\section{Triage Cancer}

https://triagecancer.org/Checklist-COVID19
Provides information about managing work, health insurance, and finances during the pandemic. 
Page 17/17 